Case Report

\title{
Fixation of the neglected subcutaneous osteochondral fragment of large medial femoral condyle defect in adult male: A case report
}

\author{
Mustafa Akkaya $^{\text {a,*, }}$ Mehmet Emin Simsek ${ }^{\mathrm{b}}$, Ceyhun Caglar ${ }^{\mathrm{c}}$, Ali Said Nazligul ${ }^{\mathrm{a}}$, \\ Serhat Akcaalan ${ }^{\mathrm{a}}$, Safa Gursoy ${ }^{\mathrm{a}}$ \\ ${ }^{a}$ Department of Orthopedics and Traumatology, Ankara Yildirim Beyazit University Faculty of Medicine, 06100, Ankara, Turkey \\ ${ }^{\mathrm{b}}$ Department of Orthopedics and Traumatology, Lokman Hekim University Faculty of Medicine, 06100, Ankara, Turkey \\ ${ }^{\mathrm{c}}$ Department of Orthopedics and Traumatology, Ankara City Hospital, 06800, Ankara, Turkey
}

\section{A R T I C L E I N F O}

\section{Keywords:}

Osteochondral fragment

Medial femoral condyle

Internal fixation

Knee

\begin{abstract}
A B S T R A C T
Knee osteochondral fractures are among the most common orthopaedic injuries, and their diagnosis and treatment is always open to debate among orthopaedic surgeons. In this case report, we described the surgical treatment and 2 years of follow-up of an adult male patient who underwent delayed osteochondral fixation with an iliac graft.

A medial parapatellar incision was made to perform open reduction and internal fixation. The fracture surface at the medial condyle of the femur was debrided with a curette, followed by placement of the tricortical graft harvested from the iliac crest into the defect at the medial femoral condyle. The osteochondral fragment was then placed on top and was fixed with three headless compression screws together with the iliac crest graft for joint surface restoration. While the patient's preoperative knee injury and osteoarthritis outcome score (KOOS) was 38 and it increased to 74 during two years follow-up.

Despite the scarcity of information on delayed fixation of osteochondral fractures, osteochondral fragments with preserved viability should be fixed, regardless of the timing, as suggested by the successful outcomes obtained in this study.
\end{abstract}

The case

- Patients diagnosed with osteochondral fractures often present with pain and limitation of movement

- The most important point for diagnosis is the suspicion of osteochondral fracture

- After the diagnosis of osteochondral fracture, appropriate fixation options should be evaluated immediately

\section{Lessons learned}

- Osteochondral fracture is a diagnosis that should be kept in mind in patients presenting after trauma

- Even if a lot of time passes after osteochondral fracture fixation, there is a chance of success

\section{Introduction}

Osteochondral fractures, which are among the common orthopaedic injuries, were first described by Milgram et al., in 1943 [1]. The mechanism of injury generally consists of impaction, avulsion, and shearing forces [2]. Patients generally have history of trauma and present with symptoms such as swelling, pain, and limited movement. Patients are generally diagnosed and receive treatment during the acute and subacute stages. However, delayed diagnosis of osteochondral fractures generally leads to the excision thereof [3] due to the limited healing ability of articular cartilage [4].

There are three potential treatment algorithms for treating ostechondral fractures [5]: (1) fixation of the osteochondral fragment, (2) excision of the osteochondral fragment, and (3) the use of regenerative procedures (bone marrow stimulation or any transplantation technique that aims for bone and cartilage regeneration) to ensure healing of the osteochondral injury [5]. Osteochondral fracture fixation is generally

Abbreviations: KOOS, Knee injury and osteoarthritis outcome score; CT, Computed tomography; MRI, Magnetic resonance imaging.

* Corresponding author. Department of Orthopaedics and Traumatology, Ankara Yildirim Beyazit University, 06100 Ankara, Turkey. Tel: +90 5325720503.

E-mail address: makkaya@outlook.com (M. Akkaya). 


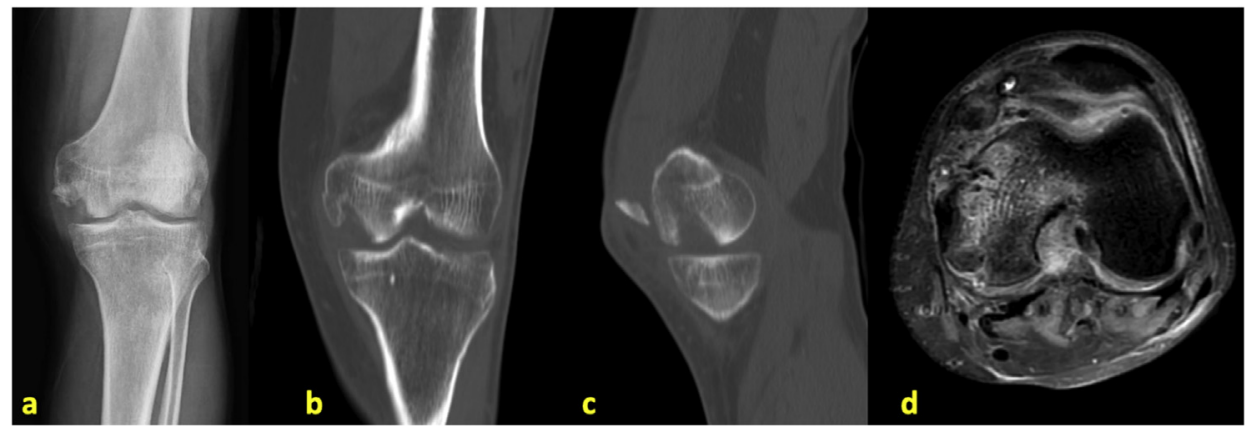

Fig. 1. Preoperative radiographic (a), CT (b and c), and MRI (d) images.

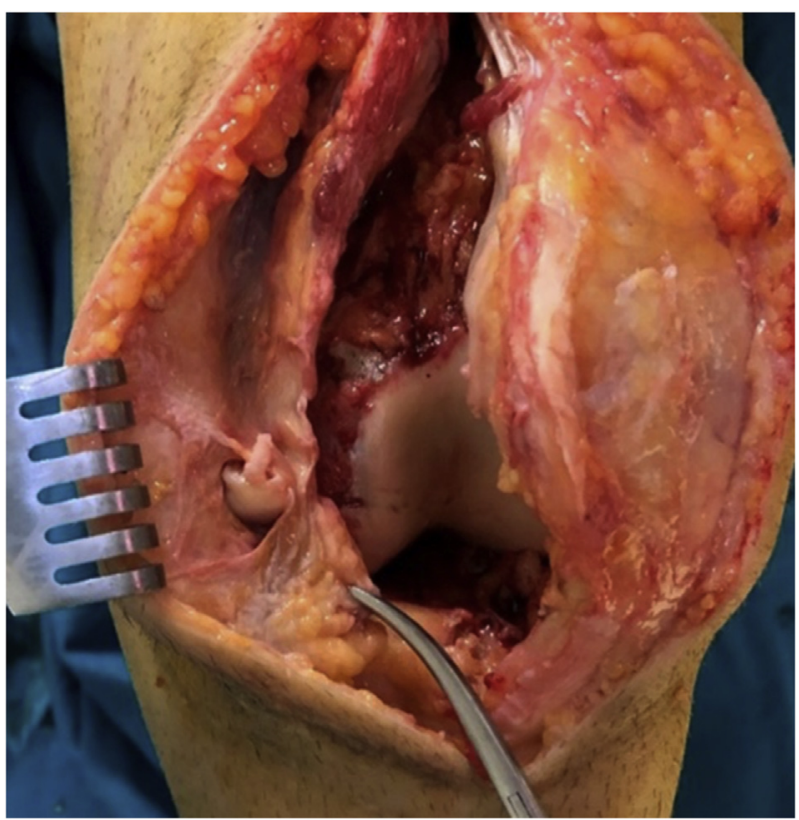

Fig. 2. Subcutaneous osteochondral fragment.

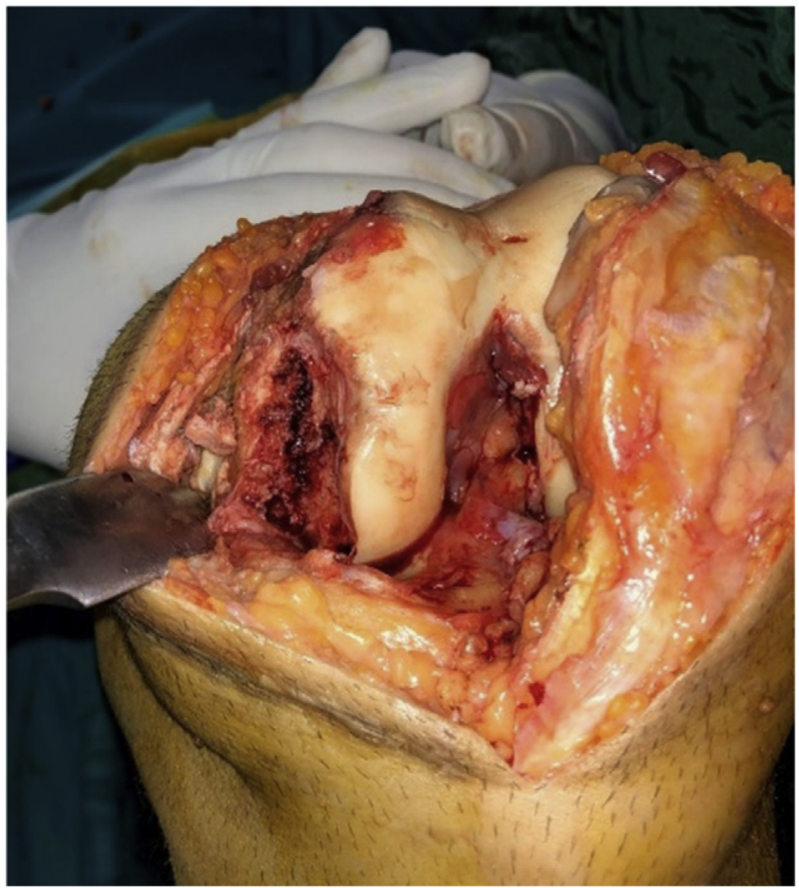

Fig. 3. Medial condyle osteochondral defect. performed during the acute phase with use of metal or bioabsorbable pins or compression screws.

In this case report, we present a patient who underwent fixation of a subcutaneous osteochondral fragment with a graft harvested from the iliac bone 3 months after trauma.

\section{Case presentation}

Here, we present the case of a 41-year-old male patient who presented to our clinic with pain and limited movement in the left knee. According to the patient's history, he had presented to the emergency department of another center with an open wound in the left knee after a traffic accident and had undergone suturation following wound debridement nearly 3 months ago. The diagnosis of an osteochondral fracture was missed, and there was no further medical intervention for the knee injury. Until the time of admission to our clinic, the knee joint pain gradually increased and progressive limitation in the range of motion of the joint occurred. Clinical examination revealed moderate swelling and pain in the left knee as well as limited joint range of motion. The patient had $15^{\circ}$ of extension loss and a range of flexion of $75^{\circ}$. He could not be mobilized without support. He had severe pain and sensitivity with palpation on the

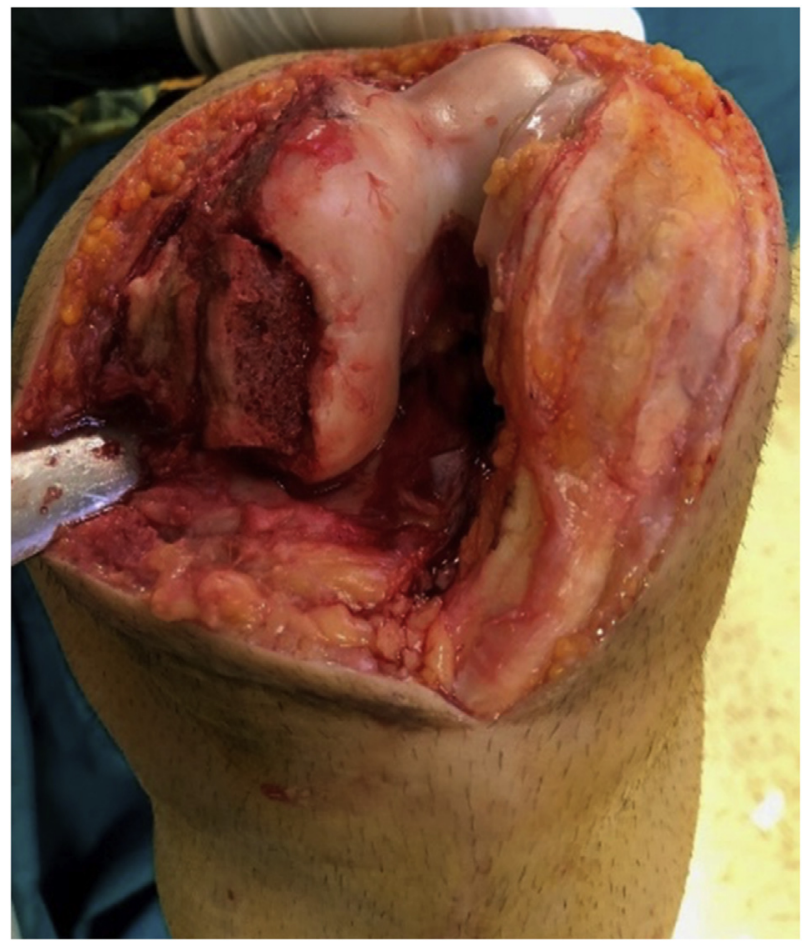

Fig. 4. Tricortical iliac graft fixation to the defect in the medial femoral condyle. 

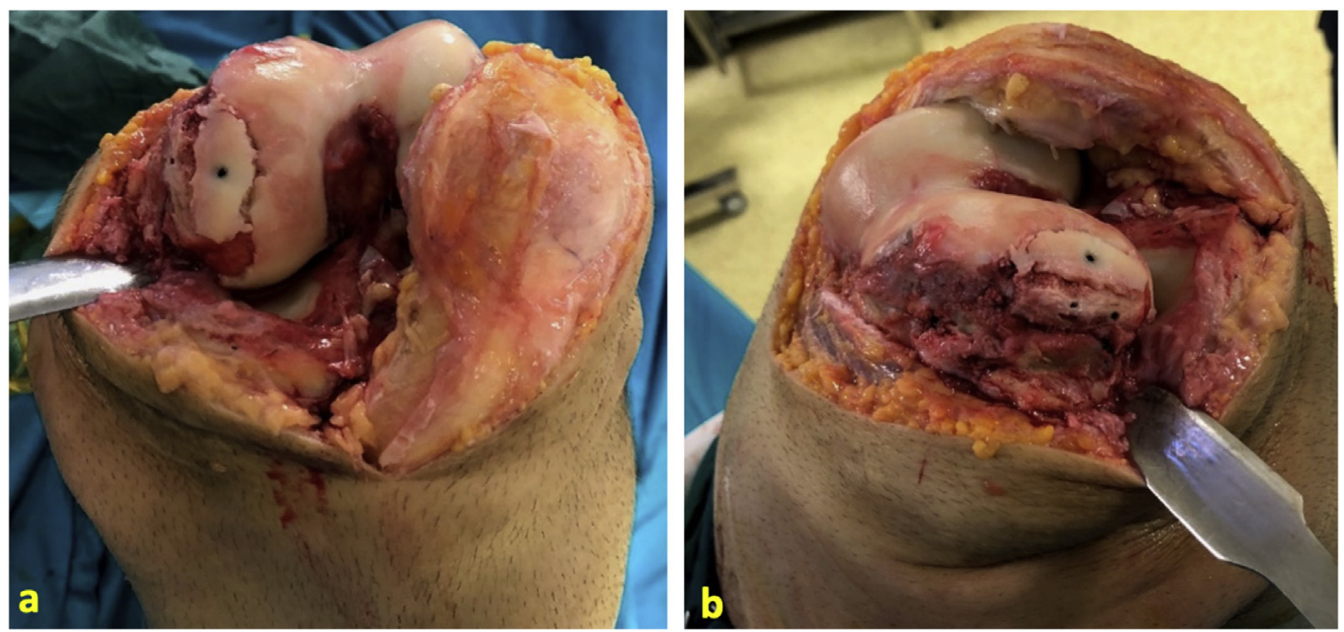

Fig. 5. Anterior (a) and lateral (b) views of the medial femoral condyle after fixation of osteochondral fragment.

medial femoral condyle of the left knee. The patient was found to have an osteochondral defect measuring nearly $5 \times 5 \mathrm{~cm}$ in the medial condyle of the left knee and a subcutaneous osteochondral fragment as seen on direct radiographs, computed tomography (CT) scans, and magnetic resonance imaging (MRI) scans (Fig. 1).

A medial parapatellar incision was made to perform open reduction and internal fixation. Once through the skin and subcutaneous tissues, the osteochondral fragment measuring nearly $5 \times 3 \mathrm{~cm}$ that had maintained supply through the fascia was reached (Fig. 2). The medial femoral condyle (Fig. 3) was reached after passing through the fascia. The fracture surface at the medial femoral condyle was debrided with a curette, followed by placement of the tricortical graft harvested from the iliac crest into the defect at the medial femoral condyle (Fig. 4). The osteochondral fragment was then placed on top and was fixed with three headless compression screws together with the iliac crest graft (Fig. 5). Joint surface restoration was found to be successful as seen on postoperative MRI scans and radiographs (Figs. 6 and 7).

During the rehabilitation program, flexion was gradually increased on a weekly basis using an angle-adjusted knee brace $\left(30^{\circ}-60^{\circ}-90^{\circ}-120^{\circ}\right)$ and weight-bearing was not allowed for 4 weeks postoperatively. Partial weight-bearing was allowed after postoperative week 4 , and full weightbearing was allowed at postoperative week 8. Running, cycling and noncontact sports were permitted after postoperative week 12 . At the time of the latest follow-up (at 2 years), the patient had returned to his daily actives and recreational sports, had no evidence of osteoarthritis on radiographs (Fig. 8) and he could reach full extansion $-120^{\circ}$ flexion knee joint range of motion and had a knee injury and osteoarthritis outcome score (KOOS) of 73.8. While calculating KOOS, the scores obtained from the parameters symptoms, pain, function, sports and recreational activities, and quality of life were calculated and averaged.

\section{Discussion}

The number of studies reporting delayed fixation of a missed osteochondral fracture in adults is very limited. It is of utmost importance to know the diagnosis and treatment methods for patients with osteochondral fractures, since this condition is very rare.

According to one study, an acute osteochondral fragment measuring $>1-1.5 \mathrm{~cm}$ that is located in the load-bearing region should be treated with open reduction and internal fixation [6]. Supporting this view, Wright et al. [7] reported that osteochondral fragment excision would yield poor results. According to Gowd et al. [8], arthroscopic fixation of an unstable osteochondral fracture of the medial femoral condyle with a nonabsorbable screw reduces morbidity and osteoarthritis progression. Similarly, Barrett et al. [9] performed internal fixation with metal screws in 22 patients with unstable osteochondral fragments in the femoral condyle and observed a satisfactory union rate and good knee function at a mean of 31 months of follow-up. Open reduction and internal fixation of the osteochondral fragment should be performed as soon as possible after diagnosis.

To our knowledge, there are two studies on delayed osteochondral fracture fixation in the literature $[10,11]$. However, one of those studies
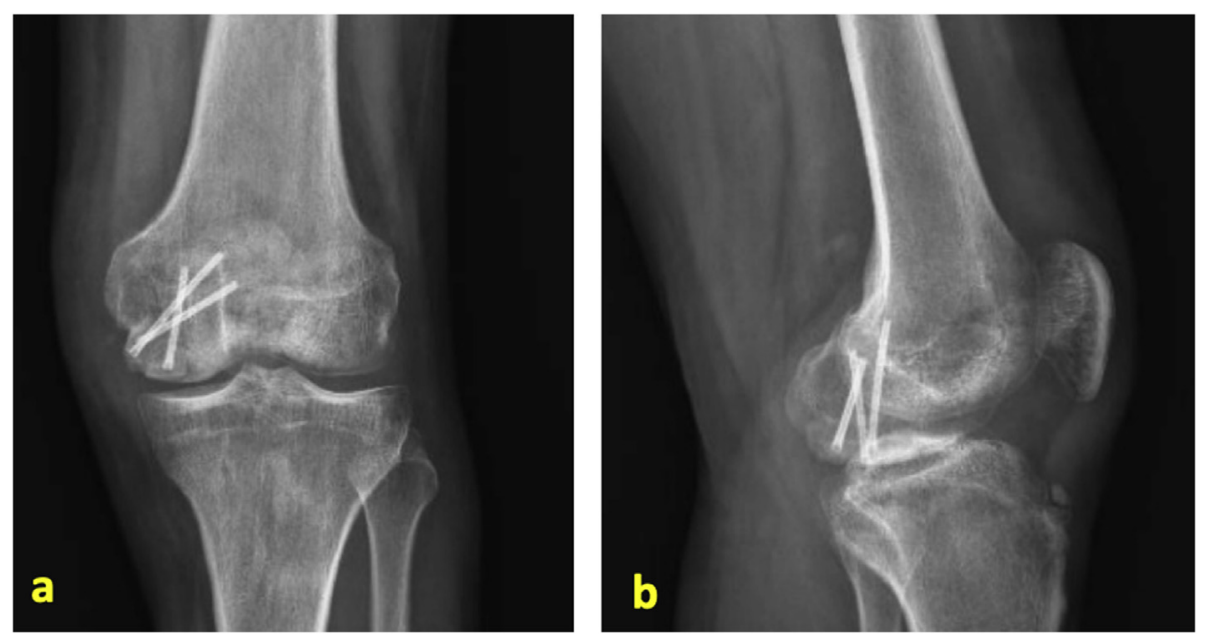

Fig. 6. Postoperative anteroposterior (a) and lateral (b) radiographs. 


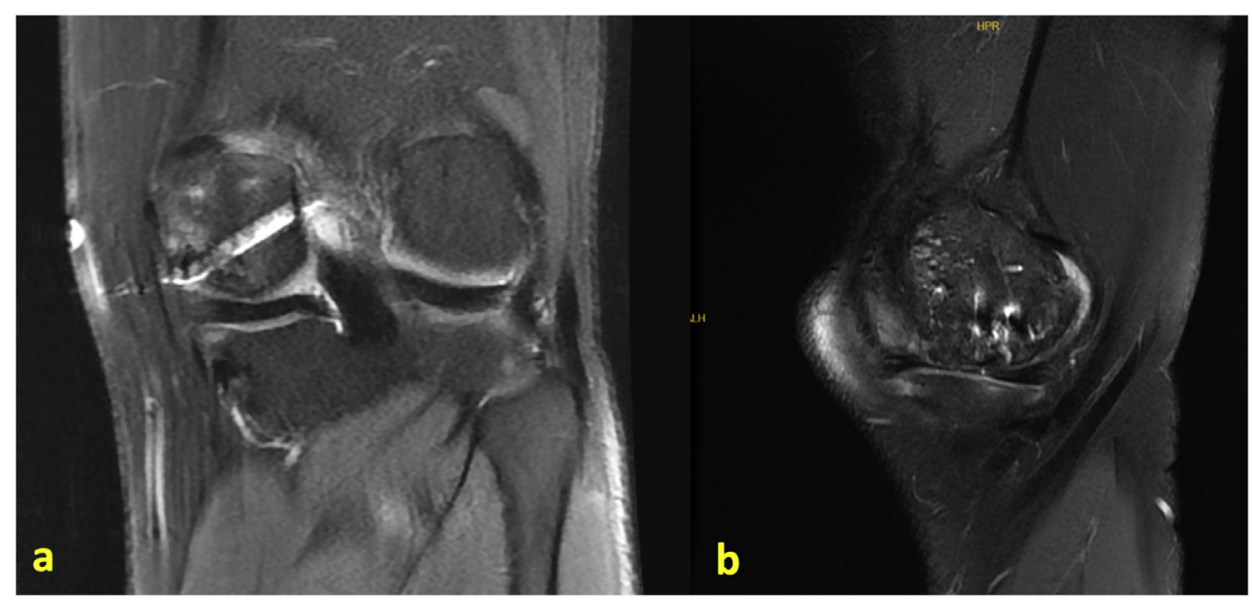

Fig. 7. Coronal (a) and sagittal (b) MRI sections made at 2 years postoperatively.

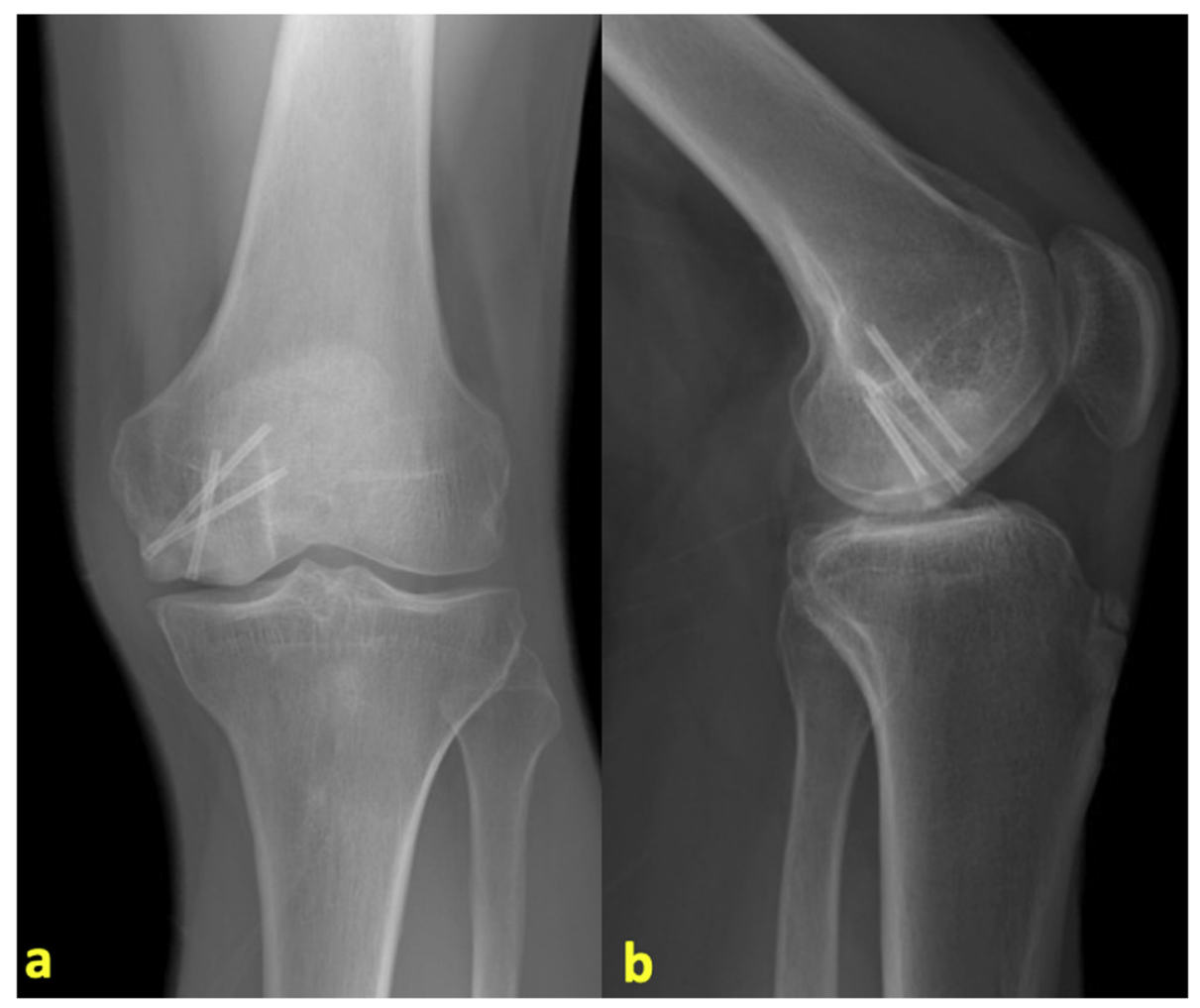

Fig. 8. Anteroposterior (a) and lateral (b) radiographs made at 2 years postoperatively.

involved the lateral femoral condyle and the other involved the patella, whereas our patient had a fracture of the medial femoral condyle. One of those studies described arthroscopy-assisted osteochondral fracture fixation in a 20-year-old male patient who had pain in the left knee for 6 months without history of trauma [10], whereas the other study described the fixation of a patellar osteochondral fracture at 8 weeks after trauma in a 23-year-old female patient [11]. The findings of those studies indicate that osteochondral fracture fixation, even if it is delayed, leads to successful clinical outcomes. Therefore, we performed open reduction and internal fixation to treat the neglected osteochondral fracture of the medial femoral condyle.

In most cases, the treatment of late-diagnosed osteochondral fractures involves excision of the osteochondral fragment [4,5]. In such cases, additional methods such as osteochondral allografting/autografting and bone marrow stimulation methods are required to prevent progression of the defect to secondary osteoarthritis, depending on defect size [12]. According to Lee and Kim [13], individual techniques are insufficient in massive and complex osteochondral lesions, and fixation of the osteochondral fragment should be combined with osteochondral autograft transplantation. In the present study, we also provided biological support for the osteochondral fragment fixation with autografting from the iliac crest in order to cover the defect located in the subchondral region and to obtain successful outcomes from delayed fixation. In the last follow-up of the patient, we observed that we could achieve the said successful outcomes.

According to the outcomes observed in the present case, there is no definitive time limit for the fixation of osteochondral fractures in adult patients when the viability of the osteochondral fragment is preserved. Primary fixation should be initially considered, even in delayed cases, if the size and integrity of the osteochondral fragment allows for primary 
fixation. Moreover, biological support with autografting should also be provided in case of existing bone defects.

\section{Conclusion}

Despite the scarcity of information on delayed fixation of osteochondral fractures, osteochondral fragments with preserved viability should be fixed, regardless of the timing, as suggested by the successful outcomes obtained in this study. Restoring the articular surface, albeit late, will allow for better clinical outcome and delay osteoarthritis progression.

\section{Declaration of competing interest}

The authors declare that they have no known competing financial interests or personal relationships that could have appeared to influence the work reported in this article.

\section{References}

[1] Milgram JE. Tangential osteochondral fracture of the patella. J Bone Joint Surg Am 1943;25(2):271-80.

[2] Farr J, Covell DJ, Lattermann C. Cartilage lesions in patellofemoral dislocations: Incidents/locations/when to treat. Sports Med Arthrosc Rev 2012;20(3):181-6. https://doi.org/10.1097/JSA.0b013e318259bc40.

[3] Kang H, Li J, Chen X-X, Wang T, Liu S-C, Li H-C. Fixation versus excision of osteochondral fractures after patellar dislocations in adolescent patients. Chinese Med J (Engl.) 2018;131(11):1296-301. https://doi.org/10.4103/03666999.232800 .
[4] Jiang S, Guo W, Tian G, Luo X, Peng L, Liu S, et al. Clinical application status of articular cartilage regeneration techniques: Tissue-engineered cartilage brings new hope. Stem Cells Int 2020:5690252. https://doi.org/10.1155/2020/5690252. 2020 Jun 30.

[5] Kühle J, Angele P, Balcarek P, Eichinger M, Feucht M, Haasper C, et al. Treatment of osteochondral fractures of the knee: a meta-analysis of available scientific evidence. Int Orthop 2013;37(12):2385-94. https://doi.org/10.1007/s00264013-2070-7.

[6] Bauer KL. Osteochondral injuries of the knee in pediatric patients. J Knee Surg 2018;31(5):382-91. https://doi.org/10.1055/s-0038-1625956.

[7] Wright RW, McLean M, Matava MJ, Shively RA. Osteochondritis dissecans of the knee: Long-term results of excision of the fragment. Clin Orthop Relat Res 2004; 424:239-43. pmid: 15241178.

[8] Gowd AK, Beck EC, Nabor D, Waterman BR. A staged arthroscopic approach to fixation of unstable osteochondritis dissecans in the medial femoral condyle of the knee using nonabsorbable fixation screws. Arthrosc Tech 2020;9(4):e477-81. https://doi.org/10.1016/j.eats.2019.11.021.

[9] Barrett I, King AH, Riester S, van Wijnen A, Levy BA, Stuart MJ, et al. Internal fixation of unstable osteochondritis dissecans in the skeletally mature knee with metal screws. Cartilage 2016;7(2):157-62. https://doi.org/10.1177/ 1947603515622662.

[10] Li R, Guo G, Chen B, Zhu L, Lin A. Arthroscopically-assisted reduction and fixation of an old osteochondral fracture of the lateral femoral condyle. Med Sci Monit 2012; 18(12):CS117-20. https://doi.org/10.12659/msm.883637.

[11] Hoshino CM, Thomas BM. Late repair of an osteochondral fracture of the patella. Orthopedics 2010;33(4). https://doi.org/10.3928/01477447-20100225-25.

[12] Pedersen ME, DaCambra MP, Jibri Z, Dhillon S, Jen H, Jomha NM. Acute osteochondral fractures in the lower extremities - approach to identification and treatment. Open Orthop J 2015;9:463-74. https://doi.org/10.2174/ 1874325001509010463.

[13] Lee BI, Kim BM. Concomitant osteochondral autograft transplantation and fixation of osteochondral fragment for treatment of a massive osteochondritis dissecans: A report of 8-year follow-up results. Knee Surg Relat Res 2015;27(4):263-8. https:// doi.org/10.5792/ksrr.2015.27.4.263. 